\title{
Model and observed seismicity represented in a two dimensional space
}

\author{
M. Ciputo (*) \\ Received on November $30 t \mathrm{~h}, 1976$
}

SUMmary. - In recent years theoretical seismology has introduced some formulae relating the mignitude and the seismic moment of earthquakes to the size of the fault and the stress drop which gencrated the earthquake.

In the present paper we introduce a model for the statisties of the carthquakes based on these formulae. The model gives formulae which show internal consistency and are also confirmed by observations.

For intermediate magnitudes the formulae reproduce also the trend of linearity of the statisties of magnitude and moment observed in all the seismic regions of the world. This linear trend ehanges into a curve with increasing slope for large magnitudes and moment.

When a catalogue of the magnitudes and/or the seismic moment of the earthquakes of a seismic region is available, the model allows to estimate the maximum magnitude possible in the region.

Riassunto. - Recentemente sono state introdotte in sismologia teorica alcume formule che legano la magnitudo ed il momento sismico dei terremoti alla dimensione della faglia ed al rilassamento di sforzo che hanno generato il terremoto stesso.

In questa nota si introduce un modello per le statistiche dei terremoti basato su quelle formule. Il modello dà formule che hanno consistenza in. terna e che sono confermate dalle osservazioni.

Per magnitudo di valore intermedio queste formule riproducono landamento lineare delle statistiche delle magnitudo edel momento osservato in tutte le regioni sismiche del mondo. Questo andamento lineare si muta in una curva di pendenza crescente colla magnitudo per grandi magnitudo e momento.

Se è disponibile un catalogo delle magnitudo e'o dei momenti sismici di una regione sismica, il modello permette di stimare la massima magnitudo possibile nella regione.

(*) Istituto di Fisica, Università di Roma. 


\section{INTRODUCTION}

The problem of developing stochastic models for earthquakes occurrence raised strong interest since the late 1950 's.

The unsatisfactory conclusion after these studies is that statistical models, based on different principles, lead to similar observed efiects because they depend on too large a number of parameters to allow the fitting of almost any data.

Inspite of the fact that there are plenty of reasons to consider earthquakes as a deterministic phenomenon, less interst was raised in the problem of developing mechanical models; we had to wait untill 1967 to have an interesting approach with a paper by Burridge and Knopofr.

At present, to proceed further it is necessary to take a deeper look at the physical aspect of the various types of process and therefore to the so called genetic models.

Interesting studies on the mechanism of stick slip have been male in the laboratory (Brune, 1973; Archuleta and Brune, 1975) (3,1) measuring particle velocities and stress drop.

A first attempt to provide a genetic statistical model for the stick slip mechanism was made by Caputo $(1975,1976 a)\left({ }^{8,7}\right)$ and led to the conclusion that the so called Ishimoto Iida (I.I., 1939) (12) empirical law can be satisfactory only in a limited magnitude range, and that even in this range, in some cases, it conld be satistactory only in first approximation; in the same paper evidence was also given that the $b$ coefficient of the I.I. law, in the intermerliate magnitude range in Europe and the Mediterranean region could be decreasing with increasing magnitude.

In subsequent papers Caputo $(1976 \mathrm{~b}, 1975)\left({ }^{5,6}\right)$ used a more exact formulation of the stress field and generalised the result also to the distribution law of the seismic moment.

Recently the problem of explaining the above mentioned empirical law has become important because of the practical implications mostly for engineering purposes. Usually one needs to decide.

1) - wether the law can actually be linear

2) - in which range it is linear

3) - what happens outside of this range, for instance wheather the slope is decreasing or increasing for small values of $M$;

4) - the possibility to predict the maximum value of magnitude, seismic moment and fault size in a given region. 
In this note these problems are considered without specifying the mechanism of the earthquakes which leads to different algebra and results than in the other notes (Caputo, 1975, 1976a, 1976b, 1977) $(5,6,7,8)$.

It is shown that the property of linearity of the frequency magnitude relation and of the frequency moment relation is independent of the mechanical model used to represent the earthquakes. The end points of the intervals where the above mentioned relations are linear are used to determine the maximum magnitude and the maximum moment possible in a seismic region when a catalogue of earthquakes of the region is available.

\section{THE MODEL}

To obtain the model let us consider that a given portion of earth's crust is crossed by a system of faults of area $S$, of linear dimension $l=S^{1 / 2}$.

In this model it is assumed that the relaxation of the elastic energy in the volume $l^{3}$ around a fault has a limited eflect on the stress distribution in the region; which is true only if the number and size of the faults is such that the total volume associated with the faults is smaller than the region considered.

This should be checked in each region where this model is applied.

We assume also that in the system of faults the stress accumulates linearly with time

$$
\varepsilon t
$$

where $\varepsilon$ is the rate of stress accumulation, that the stress drop $p$ - $p_{0}$ ( $p$ is the stress accumulated and $p_{0}$ is the residual stress) is the same for all the earthquakes of the same fault, and that the stress drops to the same value $p_{0}$ after all the earthquakes.

The value of $t$, returning period of the earthquakes of average stress drop $p-p_{0}$ on a given fault, is given by

$$
t:=\left(p-p_{0}\right) / \varepsilon
$$

where $p_{0}$ is the residual stress, which we assume to be the same after all the earthquakes. Considering in first approximation that the faults have a circular area, the formula (e.g. Caputo 1977) $\left(^{6}\right)$ 


$$
\begin{gathered}
E=10^{\beta+\gamma M}=\frac{\eta k}{2 \mu} l^{3}\left(p^{2}-\eta_{0}^{2}\right) \\
M_{0}=l^{3}\left(p-\eta_{0}\right) / c
\end{gathered}
$$

relate the magnitude $M$, the energy $E$ and the seismic moment $M_{0}$ to the linear dimension $l$, in a medium of rigidity $\mu$ for an event of stress drop $p-p_{0}, \eta$ is the factor accounting for the transformation of the energy released into elastic wares, $k$ and $c$ are geometric factors, $\beta$ and $\gamma$ are parameters relating the energy to the magnitude (e.g. see Bath 1973).

Assuming that $D l^{-1}-(l l$ is the number of faults of the system with linear dimension $l$ in the range $l, l+d l$ and that $P\left(p-p_{0}\right)^{\alpha} d p$ the number of stress drops in the range $p-p_{0}, p-p_{0}+\mathrm{d} p$, the number of earthquakes in the range $l, l+\imath l, p-p_{0}, p-p_{0}+\mathrm{d} p$ and occurred in the time interval $T$ is

$$
\frac{T P D \varepsilon}{l^{v}}\left(p-p_{0}\right)^{\alpha-1} \mathrm{~d} l \mathrm{~d} p
$$

where $t$ is the return period of the earthquakes with stress drop $p-p_{0}$.

The average stress drop could lave a frequency distribution constant for all the values of $p(\alpha=0, P=1)$, but it could also be a function of some geometric parameters, (for example of the direction between the fault and the dominating tectonic force (Caputo 1975, $1976 \mathrm{a}, 1976 \mathrm{~b}, 1977) \mathbf{( 5 , 6 , 7 , 8}^{(5,}$ therefore the integral could not be computed unless the relation between $p$ and the above mentioned parameters is known. However, as we shall see there is an interval in which the values of $M$ or $M_{0}$ can be associated to any stress drop in the range $p_{1}, p_{2}$ of variation of $p$, and the integration of [t] does not depend on $M$ or $M_{0}$. In this case we can derive some important properties of the distribution of $M$ and $M_{0}$.

In fact representing $l$ and $\gamma$ in a cartesian plane, the extremal values $\left(l_{1}, l_{2}\right)$ of $l$ and $\left(p_{1}, p_{2}\right)$ of $p$ define a rectangle; every point of this rectangle represents a possible earthquake of the morlel and the integral [4] should be computed along the curve defined by [3] and characterized by the parameters $M$ or $M_{0}$. Depending on the values of $l_{1}, l_{2}, \eta_{1}, \eta_{2}, \eta k / \mu, \beta, \gamma$ we can have two patterns for the paths of integration as indicater in the Fig. 1a and Fig. $1 \mathrm{~b}$, in relation to the crossings of these curves with the lines $p=p_{1}$ and $p=p_{2}$. The most realistic situation is that of Fig. la. 
A direct integration of formula [4] gives the cumulative distribution functions $n(M)$ and $n_{0}\left(M_{0}\right)$; from these one may then obtain
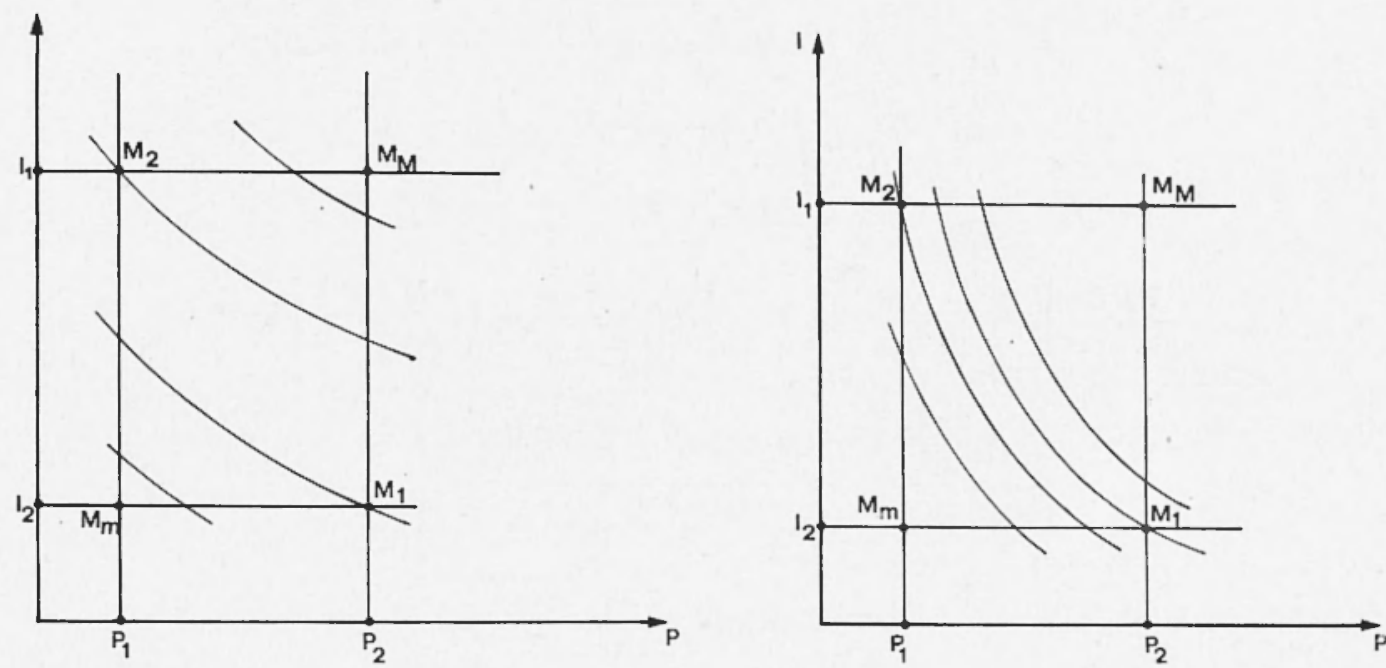

Fig $1-(a)$ and (b): Paths of integration for the computation of the integral (4).

the frequency distribution function $\bar{n}(M)=-\frac{\mathrm{d} n}{\mathrm{~d} H}$, $\bar{n}_{\mathrm{o}}\left(M_{0}\right)=-\frac{\overline{\mathrm{L}}_{\mathrm{0}}}{\mathrm{d} \boldsymbol{I}_{0}}$. They have different expressions depending on the values

$$
\begin{aligned}
& M_{1}=\frac{1}{\gamma}\left[\log \frac{\eta k}{2 \mu} l_{1}{ }^{3}\left(\mu_{2}{ }^{2}-p_{0}{ }^{2}\right)-\beta\right] ; M_{2}=\frac{1}{\gamma} \mid \log \frac{\eta l_{2} l_{2}}{2 \mu}\left(p_{1}^{2}+\right. \\
& \left.\left.-p_{0}{ }^{2}\right)-\beta\right] \\
& M_{01}=l_{1}^{3}\left(p_{2}-p_{0}\right) / c \quad M_{\mathrm{o} 2}=7_{2}{ }^{3}\left(p_{1}-p_{0}\right) / c
\end{aligned}
$$

and on the maximum $\left(I_{0.1}, Y_{v}\right)$ and minimum $\left(M_{o m}, M_{m}\right)$ values of $M$ o and $M$.

When $M_{1}<H_{2}$ and $M_{01}<M_{0^{\circ}}$ these values, called corner magnitude and corner seismic moment respectively, define the interval in which $\bar{n}(M)$ and $\vec{n}_{0}(M)$ are linear.

A direct integration of [4] when $M_{1}<M_{2}$ (or $M_{01}<M_{02}$ ) for $H_{m}<M<M_{1}$ and $M_{0 m}<H_{0}<M_{01}$ gives 


$$
\begin{aligned}
& n=\frac{T P D_{\varepsilon}}{1-v}\left\{\left[\frac{\left(p-p_{0}\right)^{\alpha}}{\alpha}\right]^{p_{2}} l_{2}^{1-v}-\left[\frac{\left(p-p_{0}\right)^{a}}{\alpha}\right]^{p_{2}} l_{1}^{1-\gamma}+\right. \\
& \sqrt{\frac{R^{3}}{l_{1}{ }^{3}+p_{0}{ }^{2}}} \quad \sqrt{\frac{R^{3}}{l_{1}{ }^{3}}+p_{0}{ }^{2}} \\
& \left.-R^{1-v} \int_{p_{1}}^{l_{1}^{3}} \frac{\left(p-p_{0}\right)^{\alpha-1}}{\left(p^{2}-p_{0}^{2}\right)^{\frac{1-v}{3}} \mathrm{~d} p}\right\} \\
& n_{0}=\frac{T P D \varepsilon}{1-v}\left\{\left[\frac{\left(p-p_{0}\right)^{\alpha}}{\alpha}\right]_{p_{1}}^{p_{2}} l_{2^{1-v}}-\left\lfloor\frac{\left(p_{2}-p_{\mathrm{o}}\right)^{\alpha}}{\alpha}-\left(\frac{c M_{\mathrm{o}}}{l_{1^{3}}}\right)^{\alpha} \frac{1}{\alpha}\right] l_{1}^{1-v}+\right. \\
& \left.-\frac{\left(\frac{c M_{0}}{l_{1}^{3}}\right)^{\alpha}-\left(p_{1}-p_{0}\right)^{-\frac{1-v}{3}}\left(c M_{0}\right)^{\frac{1-v}{3}}}{\alpha-\frac{1-v}{3}}\right\} \\
& \bar{n}=\frac{\gamma T P D \varepsilon \ln 10}{3(1-v)} \overline{i i}_{i_{1}} \frac{\left(p-p_{0}\right)^{\alpha-1}}{\left(p^{2}-p_{0}{ }^{2}\right)^{\frac{1-v}{3}}} \mathrm{~d} p \\
& \bar{n}_{0}=\frac{T P D \varepsilon}{3 \alpha-1+\nu}\left\{\frac{c^{\alpha} M_{0}^{\alpha-1}}{l^{3 c-1+\nu}}+\left(p_{1}-p_{0}\right) \ddot{a-\frac{1-\nu}{3}} e^{\frac{1-\nu}{3}} M_{0}-\frac{2+\nu}{3}\right\} \\
& R^{3}=\frac{2 \mu 10^{\beta+\vartheta} M I}{\eta k}
\end{aligned}
$$

For $M_{1}<M<M_{0}$ and $M_{01}<M_{0}<M_{02}$ we have

$$
\begin{aligned}
& n=\frac{T P D \varepsilon}{1-\nu}\left\{\gamma_{2} 1-v\left[\frac{\left(p-p_{0}\right)^{\alpha}}{\alpha}\right]_{p_{1}}^{p_{2}}-R^{1-v} \int_{p_{1}}^{p^{p_{2}}} \frac{\left(p-p_{0}\right)^{\alpha-1}}{\left(p^{2}-p_{0}\right)^{\frac{1-\nu}{3}}} d p\right\} \\
& n_{0}=\frac{T P D \varepsilon}{1-\nu}\left\{l _ { 2 } ^ { 1 - \nu } \left[\left.\frac{\left(p-p_{0}\right)^{\alpha}}{\alpha}\right|_{p_{1}} ^{p_{2}}-\left(c M_{0}\right)^{\frac{1-v}{3}}\left[\left.\frac{\left(p-p_{0}\right)^{--\frac{1-v}{3}}}{\alpha-\frac{1-\nu}{3}}\right|_{p_{1}} ^{p_{2}}\right\}\right.\right.
\end{aligned}
$$

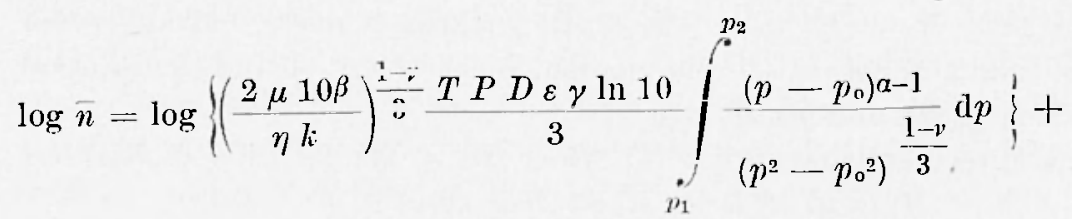




$$
\begin{gathered}
-\frac{v-1}{3} \gamma M \\
\log \bar{n}_{\mathrm{o}}=\log \left\{\frac { T P D \varepsilon c ^ { \frac { 1 - v } { 3 } } } { 3 a + v - 1 } \left[\left(p-p_{0}\right)^{\left.\left.\alpha+\frac{\nu-1}{3}\right]_{p_{1}}^{p_{2}}\right\}-\frac{2+v}{3} \log M_{\mathrm{o}}}\right.\right.
\end{gathered}
$$

For $M_{2}<M<M_{M}, M_{02}<M_{0}<M_{0 . M}$ we have

$$
\begin{aligned}
& n=\frac{T P D \varepsilon}{\alpha}\left\{\frac{\left(p_{2}-p_{0}\right)^{\alpha}}{1-v}\left(l_{2}{ }^{1-v}-\left(\frac{R}{\left(p_{2}{ }^{2}-p_{0}\right)^{1} / 3}\right)^{1-p}\right\}+\right. \\
& -R^{1-v} \int^{l_{2} / R} u^{-v}\left(\sqrt{\left.\frac{1}{u^{3}}+p_{\mathrm{o}}^{2}+p_{\mathrm{o}}\right)^{\alpha} \mathrm{d} u}\right\} \\
& \frac{1}{\left(p^{2}{ }_{2}-p^{2}{ }^{2}\right)^{1 / 3}} \\
& n_{\mathrm{o}}=T P D \varepsilon\left\{\frac{\left(p_{2}-p_{0}\right)^{\alpha} l_{2^{1-\nu}}}{\alpha(1-\nu)}+\frac{3\left(c M_{0}\right)^{\frac{1-\nu}{3}}}{(1-v)(3 \alpha-\nu+1)}+\right. \\
& +\frac{\left.\left(c M_{0}\right)^{\alpha l_{2}^{-3 a-\gamma+1}}\right)}{\alpha(3 \alpha+\gamma-1) !} \\
& \vec{n}=\frac{T P D \varepsilon \gamma \ln 10}{3} R^{1-\nu} \int_{\sqrt{\frac{R^{3}}{l_{2}{ }^{3}}+p_{0}{ }^{2}}}^{p_{2}} \frac{\left(p-p_{0}\right)^{a-1}}{\left(p^{2}-p_{0}\right)^{\frac{1-\gamma}{3}}} \mathrm{~d} p \\
& \vec{n}_{0}=\frac{T P D \varepsilon}{3 \alpha+\nu-1}\left\{-l_{2}^{-3 \alpha-\nu+1} M_{0}{ }^{\alpha-1}+\left(p_{2}-p_{0}\right)^{n-\frac{\nu-1}{3}}\right. \\
& \left.c^{\frac{1-v}{3}} M_{0}{ }^{-\frac{2+y^{\prime}}{3}}\right\}
\end{aligned}
$$

In the respective intervals where they are linear the distribution function of $M$ and $M_{0}$ are independent of the distribution function of the average stress drop; therefore they could be applied to a wide class of earthquakes mechanisms.

It results also that the slopes $\bar{B}_{2}$ and $\overline{B_{02}}$ of $\log \bar{n}(M I)$ and $\log$ $\bar{n}_{0}\left(M_{0}\right)$ in their linear regions are related by

$$
\left.\bar{B}_{2}=\bar{B}_{02}+1\right) \gamma
$$

Formula [9] does not depend on the values of $p_{0}, \alpha, v$. 
THE MAXIUA VALUES OF M AND $M_{0}$

The values of $M_{M}$ and $I_{0 M}$ of the seismic regions are of great interest in many problems, they can be obtained by fitting formulae [8] to the catalogue of earthquakes of each region.

The direct information which we have on the distribution function of the fault size is very scarse; the information on the distribution of the stress (lrop is almost nil. It is therefore reasonable to assume in the first instance that the rlistribution of stress drop is homogeneous $(P=-1, a=0)$.

Also during each earthquake the stress may or may not (lrop) to zero or, during the faulting, the fault may overshoot the equilibrium position and build a stress of sign opposite to that which generated the earthquake, as in the case of reverse faulting; it is therefore reasonable to assume the arerage value $\eta_{0}=0$.

To obtain the maximum values of $M$ and $M_{0}$, first we obtain $v$ from the linear part of $\bar{n}(M)$ and $\bar{n}_{\mathrm{o}}\left(M_{\mathrm{o}}\right)$, then with the available values of $\bar{n}(M)$ and $\bar{n}_{0}\left(M_{0}\right)$ for $M>I_{2}$ and $\Pi_{0}>M_{02}$ and by using the least square method, we obtain the parameters $\bar{A}_{3}, \bar{B}_{3}, \bar{A}_{\text {o3 }}$ and $\bar{B}_{\text {o3 }}$ delined as follows from [8]

$$
\begin{aligned}
& n_{0}\left(M_{0}\right)=T D \varepsilon\left\{\frac{l_{2}^{1-v}}{(1-v) M_{0}}+\frac{\left(\frac{p_{2}}{c}\right) \frac{v-1}{3}}{(v-1) M_{0} \frac{\nu+2}{3}}\right\} \\
& =-\bar{A}_{03} M_{0}^{-1}+\bar{B}_{03} M_{0}
\end{aligned}
$$

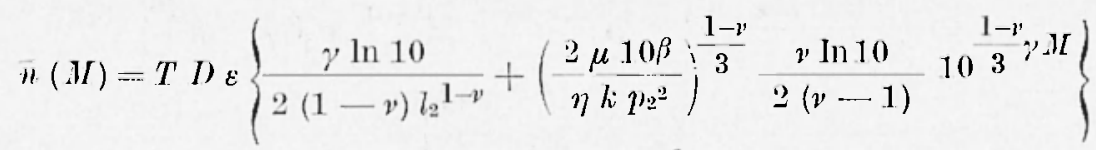

$$
\begin{aligned}
& =-\Lambda_{3}+B_{3} 10^{\frac{1-v}{3} \gamma M}
\end{aligned}
$$

In turn the computed values of $A_{33}, B_{3}, A_{03}, B_{03}$ together with [10] give a system of $t$ equations in the parameters $l_{2}, \gamma_{2}, \gamma, T D \varepsilon$.

Finally, the adjusted ralues of $A_{3}, B_{3}, A_{03}$ and $B_{03}$ give

$$
\begin{gathered}
\log M_{\mathrm{o} M}=\left(\frac{1-v}{3}\right)^{-1} \log \frac{\bar{A}_{\mathrm{o} 3}}{\bar{B}_{03}} \\
M_{M}=\left(\frac{1-v}{3} \gamma\right)^{-1} \log \frac{\bar{A}_{3}}{\bar{B}_{3}}
\end{gathered}
$$


We note also that $\gamma=\frac{2 \bar{A}_{3}}{\bar{A}_{03} \ln 10}, p_{2}=\frac{2 \mu 10^{\beta}}{c \eta k}\left(\frac{2 \bar{B}_{03}}{\gamma \bar{B}_{3} \ln 10}\right)^{\frac{3}{v-1}}$

It is obvious that, when the data on the seismic moment is not available, as it is the case in most circumstances, the second formula of [11] and [10] give directly $M_{x}$ and also $l_{2}$ when $\psi_{2}, \eta l, c, \hat{\rho}, \gamma$ are known.

An estimate of $M_{M}, M_{0 . M}$ and $l_{2}$ can be marle in a simple manner also when $p_{2}, M_{2}, Y_{02}$ are available. In fact considering that $l_{2}$ and $p_{2}-p_{0}$ are the maximum fault size and the maximum stress drop of a given region, then we have for the minimum and maximum magniturle

$$
\begin{aligned}
& M_{m}=\frac{1}{\gamma}\left[\log \frac{\eta F}{2 \mu} l_{1}{ }^{3}\left(p_{1}{ }^{2}-\eta_{0}{ }^{2}\right)-\beta\right] \\
& M_{M}=\frac{1}{\gamma}\left[\log \frac{\eta k}{2 \mu} l_{2}{ }^{3}\left(p_{2}{ }^{2}-p_{0}{ }^{2}\right)-\beta\right]
\end{aligned}
$$

We also obtain for the maximum and minimum moment

$$
\begin{aligned}
& M_{\mathrm{o} . M}=l_{2^{3}}\left(p_{2}-p_{0}\right) / c \\
& M_{\mathrm{om}}=l_{1}^{3}\left(p_{1}-p_{\mathrm{o}}\right) / c
\end{aligned}
$$

By eliminating $p_{1}, M_{m}, \Lambda_{1}, M_{\mathrm{om}}, M_{\mathrm{ol}}$, in [5], [12] and [13] we obtain the maximum values of $M$ and $M_{0}$, for a given region as function of $\eta_{2}$ when the values of $M_{2}$ and $M_{02}$ are determined from catalogues ther are

$$
\begin{gathered}
l_{2}=\left(\frac{M_{02}^{2} \eta k c^{2}}{2 \mu 10^{\beta+\gamma M_{2}}}\right)^{1 / 3} \\
M_{M}=\frac{1}{\gamma}\left[\log \frac{c^{2} \eta^{2} k^{2} M_{\mathrm{o} 2}-\eta_{2}^{2}}{4 u^{2} 10^{\beta+\gamma I_{2}}}-\beta\right] \\
M_{0 M}-\frac{c \eta k M_{\mathrm{o} 2^{2}} \eta_{2}}{2 \mu 10^{\beta+\gamma M_{2}}}
\end{gathered}
$$

It is to be noted that the maximum fault size is rletermined by the corner magnitude and corner moment only.

\section{EXPERIMENTAL CHECKS}

In this section we shall check the formulae obtained in this note with the data obtained from the catalogues of the earthquakes.

Formulae [7], assuming $a=p_{0}=0$ and $P=1$, can be written, neglecting higer order terms 


$$
\begin{aligned}
& \log n(M)=\bar{A}_{2}+\bar{B}_{2} M, \bar{B}_{2}=\frac{\gamma(1-v)}{3} \\
& \bar{A}_{2}=\log \left\{\frac{\ln 10 T D \varepsilon \gamma}{2(\nu-1)}\left(\frac{2 \mu 10 \beta}{\eta k}\right)^{\frac{1-\gamma}{3}} p_{3} \frac{2}{3}(\nu-1)\right\} \\
& \log n(M)=A_{2}+B_{2} M, \bar{B}_{2}=B_{3}, \\
& A_{2}=\bar{A}_{2}-\log \ln 10-\log \frac{\nu-1}{3} \gamma \\
& \log \bar{n}_{0}\left(M_{0}\right)=\bar{A}_{02}+\bar{B}_{02} \log M_{0}, \bar{B}_{02}=-\frac{\nu+2}{3} \\
& A_{02}=\bar{A}_{02}-\log \frac{\nu-1}{3}=A_{2}+\frac{\nu-1}{3} \log \left(\frac{2 \mu 10 \beta}{c \eta k p_{2}}\right)-\log \frac{1}{2} \\
& \log n_{0}\left(M_{0}\right)=A_{02}+B_{02} \log M_{0}, B_{02}=\bar{B}_{02}+1=-\frac{v-1}{3}
\end{aligned}
$$

For what is concerning the non linear part of $\bar{n}(M)$ and $\bar{n}\left(M_{0}\right)$ we obtain from formulae [11] and [15]

$$
\begin{gathered}
\bar{B}_{03}=10^{A_{02}}=\frac{\nu-1}{3} 10^{A_{02}}=\frac{T D \varepsilon}{\nu-1}\left(\frac{p_{2}}{c}\right)^{\frac{\nu-1}{3}} \\
\bar{B}_{3}=\frac{\hat{\imath}}{3}(\nu-1) 10^{A_{2}} \ln 10=\frac{T D \varepsilon \gamma \ln 10}{2(\nu-1)}\left(\frac{2 \mu 10 \beta}{\eta p_{2}{ }^{2}}\right)^{\frac{1-\nu}{3}} \\
\bar{A}_{3}=\bar{A}_{03} \gamma \frac{\ln 10}{2}=\gamma \frac{T D \varepsilon l_{2}{ }^{1-\nu} \ln 10}{2(\nu-1)}
\end{gathered}
$$

By combining formulae [5], [11], [12], [13], [15] and [16] we obtain

$$
\begin{aligned}
& \gamma M_{M}=\frac{3}{\nu-1}\left[\log \left(\frac{\gamma(\nu-1) \ln 10}{3 \bar{A}_{3}}\right)+A_{2}\right] \\
& \log M_{\mathrm{oM}}=\frac{3}{v-1}\left[\log \frac{\gamma(v-1) \ln 10}{6 \bar{A}_{3}}+A_{\mathrm{o} 2}\right] \\
& p_{2}=\frac{9, \cdot}{c \eta \bar{i}} 10\left(A_{2}+\log z-A_{02}\right) \frac{3}{\eta-1}+\beta \\
& l_{2}=\left(\frac{\gamma(\nu-1) \ln 10}{6 A_{3}}\right)^{1-\nu}\left(\frac{a^{2} \eta k}{2 \mu 10 \beta}\right)^{1 / 3} 10^{\left(2 \lambda_{02}-A_{2}-\log 2\right)^{\frac{1}{p-1}}} \\
& T D_{3}=\frac{(\nu-1)^{2}}{3}\left(\frac{c^{2} \eta k}{\because \mu 10 \beta}\right)^{\frac{\nu-1}{3}} 10^{2 A_{02}-A_{2}-\log 2}
\end{aligned}
$$


From world wide datum, Gutemberg and Richter (1954) (10) obtain $A_{2}=7.66, B_{2}=-0.93$, and Chinnery and North (1975) $\left(^{\circ}\right)$ obtain $A_{\mathrm{o} 2}=17.47, B_{\mathrm{o} 2}=-0.61$.

From the values of $B_{0}$ and $B_{02}$ we obtain $\nu=2.83$ and $\gamma=1.52$. The value of $\gamma$ is also obtained eliminating $l$ in formulae [3] (as it was done in Caputo (1977) $\left(^{8}\right)$ to correlate linearly the values of $M$ and $M_{0}$ of Chinnery and North $(1975)\left({ }^{9}\right)$; we find $\gamma=1.55$ ln very good agreement with the other value.

From a best fit to the datum of Gutemberg and Richter (1954) ( $\left.{ }^{10}\right)$ for large values of $M$ we tentatively obtain $\bar{A}_{3}=0.4$.

would tentatively give $M_{M}=8.7$.

The known value of $A_{\text {o2 }}$ (Chinnery and North, 1975) ( $\left.{ }^{\circ}\right)$, substituted in formulae [17] with the values of $A_{2}$ and $A_{3}$ and assuming $\mu=3.10^{11}$ and reasonable values for $\eta, k, c$, give indicative values of $l_{2}, p_{2}, M_{M}, M_{\mathrm{o} . M}$ and $D T \varepsilon$ which are satisfactory; better values for all these parameters could be obtained if the data come from a single seismic region; the data analysed above do not satisfy this requirement. It is also possible that the empirical relation between $M$ and $M_{0}$ used by Chinnery and North $(1975)\left({ }^{9}\right)$ to obtain $A_{02}$ was not completely adequate as suggested by Caputo $(1977)\left({ }^{6}\right)$ or that the datum used was not sufficient.

\section{Coxclustoxs}

The model presented here to represent the statistical data on earthquakes which are available today fits satisfactorly the data of most seismic regions of the world. Also, if a catalogue of the magnitudes and/or seismic moments of a seismic region are available, the formulae enable to estimate the maximum magnitude and the maximum moment possible and the distribution of the faults in the region.

The two hypothesis on the distribution of the linear dimensions of the faults and of the distribution of the stress drops cannot be verified experimentally at the moment. However it seems reasonable to accept that the number of faults is a decreasing function of their size and that the number of stress drops is also a decreasing function of the stress drop. 


\section{REFERENCES}

(1) ArCitleta R. J., BRuse l. N., 1975. - Surface strong motion associaled with a slick slip event in a foom rubber model of earthquakes. "Bull. Scisn. Soc. Am. ${ }^{\circ}, 65,5$, pp. 1053-1071.

$\left({ }^{2}\right)$ BAтті II., 1973. - Introduction to seismology. Birkhauser-Verlag, Basel.

(3) BRUne J. N., 1973. - Earthqualie modeling by stick-slip along precut surfaces in stressed foam rubber. "Bull. Seism. Soc. Am.", 63, 6, pp. $2105-2119$.

(1) BURRIDGE R. and KNoporf L., 1967. - Model and theoretical seismicity. "Bull. Seismol. Soc. Amer.", 57, pp. 341-371.

$\left(^{5}\right)$ Caputo M., 1976b. - Mechanical models for the statistics of earthqualies magnitude, moment and fault distribution with stress drop to zero. Rendic. Acc. Naz. Lincei, Mem. (1. Sc. Mat. Fis. Nat. (In press).

$\left({ }^{6}\right)$ CapuTo $11 ., 1977$. -1 mechanical model for the stalistics of earthquales magnitude moment and fault distribution. "Bull. Seism. Soc. Am." June.

(7) CAputo MI., 1976a. - Properties of eartlqualies statisties. "Amnali Matematica", $111,185$.

$\left.{ }^{8}\right)$ CAPUTO M., 1975. - Mechanical models of earthquales and their slatisties. Proceedings F.S.C. Symposium on earthquake risk for nuclear power plant.

(') ('IINNery II. A., Nortir R. G., 1975. - The frequence of very large earthquakes. "Science", pp. 1197-1198, 19 dec. 1975.

(10) Gutembera B., Richter (C. F., 1954. -- Seismicity of the Earth and associated phenomena. Princeton Liniv. Press, Princetion N.Y.

(11) Kanamori II., ANDERson Don I., 1975. - Theoretical basis of some empirical relations in Seismology. "Bull. Scism. Soe. $\Lambda$ m.", 65, 5, pp. 1073-1095.

(12) Ismmoto M., IIDA K., 1939. - Bull. Earth. Res. Inst., 17, pp. 443-478. 